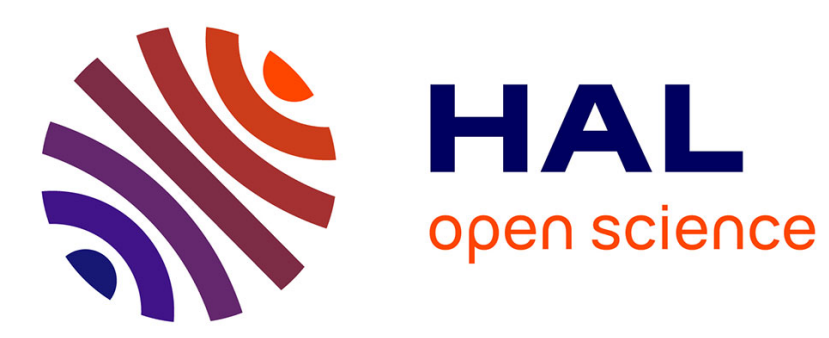

\title{
Finite time stability of differential inclusions
}

\author{
Emmanuel Moulay, W. Perruquetti
}

\section{To cite this version:}

Emmanuel Moulay, W. Perruquetti. Finite time stability of differential inclusions. IMA Journal of Mathematical Control and Information, 2005, 22 (4), pp.465-475. 10.1093/imamci/dni039 . hal03088744

\section{HAL Id: hal-03088744 https://hal.science/hal-03088744}

Submitted on 19 Jan 2021

HAL is a multi-disciplinary open access archive for the deposit and dissemination of scientific research documents, whether they are published or not. The documents may come from teaching and research institutions in France or abroad, or from public or private research centers.
L'archive ouverte pluridisciplinaire HAL, est destinée au dépôt et à la diffusion de documents scientifiques de niveau recherche, publiés ou non, émanant des établissements d'enseignement et de recherche français ou étrangers, des laboratoires publics ou privés. 


\title{
Finite time stability of differential inclusions
}

\author{
E. MOULAY* ${ }^{* \dagger}$ W. PERRUQUETTI ${ }^{\dagger}$
}

May 20, 2005

\begin{abstract}
In this paper, the problem of finite time stability is investigated for differential inclusion. Two sufficient conditions for finite time stability, using a smooth Lyapunov function and a nonsmooth one, are established. Then, the same idea is used to give two necessary conditions. Examples are developed using the concept of Krasovskii solutions for differential equation with discontinuous righthand sides.
\end{abstract}

\section{Introduction}

The stability properties of non linear systems has been developped over the last century. Nowadays, mathematicians and engineers have to face some more precise time specifications of the behavior of the state variables (or outputs) for real processes. This is one of the reason why the finite time stability has been developed, essentially for differential equations. In fact, the attraction of the motions to the desired target (in general an equilibrium point) takes place in a finite time.

The problem of finite time stability for differential inclusions is a natural question which arises when studying finite time stabilization using discontinuous feedback. Indeed much systems are not continuously stabilizable and one has recourse to discontinuous controller. Finite time stabilization is often better than asymptotic stabilization because solutions reach the equilibrium and the time of convergence can be pre-specified. Some researches on finite time stabilization for systems described by ordinary differential equations, as mechanical systems, gives interesting results (see (Hong 2002)).

The aim of this article is to investigate the finite time stability for differential inclusions using Lyapunov functions. Some results have already appeared on finite time stability of differential inclusion (see (Perruquetti \& Drakunov 2000) and (Orlov 2003)). As regards ordinary differential equations, the first result

\footnotetext{
*Laboratoire Paul Painlevé (UMR CNRS 8524), UFR de Mathématiques, Université des Sciences et Technologies de Lille, 59655 Villeneuve d'Ascq Cedex; E-mail: emmanuel.moulay@ec-lille.fr

${ }^{\dagger}$ LAGIS (CNRS UMR 8021), Ecole centrale de Lille, BP 48, Cité Scientifique, 59651 Villeneuve d'Ascq Cedex; E-mail: wilfrid.perruquetti@ec-lille.fr
} 
using smooth Lyapunov functions comes from Haimo in (Haimo 1986). It has been extended to nonsmooth Lyapunov functions in (Bhat \& Bernstein 2000) and to non autonomous systems in (Moulay \& Perruquetti 2003). The main idea was to use an increasing condition on a Lyapunov function to obtain the finite time convergence. Here, the same idea is taking up and developing for differential inclusions.

The paper is organized as follows. After some notations in sections 2 , the definitions and concept of finite time stability are given for differential inclusions in section 3. Then in section 4, one uses first an increase on a smooth Lyapunov function then on a nonsmooth one to provide two sufficient conditions for finite time stability of differential inclusions. Finally in section 5, the first increase is inverting in order to give two necessary conditions for finite time stability of differential inclusions. Moreover, using the concept of Krasovskii solutions, we examine some examples of finite time stable systems with discontinuous righthand sides. Indeed, this is one of the main applications of differential inclusions.

\section{Notations}

Throughout the paper $\mathcal{X}$ will denote a non empty open subset of $\mathbb{R}^{n}, \mathcal{V}$ a neighborhood of the origin in $\mathcal{X}, \mathcal{B}$ the unit open ball of $\mathbb{R}^{n}$ and $\|\cdot\|$ the euclidian norm of $\mathbb{R}^{n}$. The smallest closed convex set containing the set $\mathcal{A}$ is denoted by $\overline{c o}(\mathcal{A})$. Let us recall the definition given in (Aubin \& Cellina 1984): a set valued function $F$ on the vector space $\mathcal{E}$ to the vector space $\mathcal{G}$ is a function that maps $x \in \mathcal{E}$ to a subset $F(x)$ of $\mathcal{G}$. $F$ is upper semi-continuous (u.s.c) if for all $x \in \mathcal{E}$ and $\epsilon>0$, there exists $\delta>0$ such that

$$
\|x-y\|_{\mathcal{E}}<\delta \Rightarrow F(y) \subset F(x)+\epsilon \mathcal{B}^{\mathcal{G}}
$$

where $\mathcal{B}^{\mathcal{G}}$ is the open unit ball in $\mathcal{G}$, and $F$ is Lipschitzean if there exists a constant $l \geq 0$ such that for all $x, x^{\prime} \in \mathcal{E}$,

$$
F(x) \subset F\left(x^{\prime}\right)+l\left\|x-x^{\prime}\right\|_{\mathcal{E}} \mathcal{B}^{\mathcal{G}} .
$$

A function $x:[a, b] \rightarrow \mathbb{R}^{n}$ is absolutely continuous if for all $\epsilon>0$, there exists $\delta>0$ such that, for any countable collection of disjoint subintervals $\left[a_{k}, b_{k}\right] \subset$ $[a, b]$ such that $\sum_{k}\left(b_{k}-a_{k}\right)<\delta$, we have $\sum_{k}\left\|x\left(b_{k}\right)-x\left(a_{k}\right)\right\|<\epsilon$.

The upper and lower right directional Dini derivatives of a function $f$ : $\mathbb{R}^{n} \rightarrow \mathbb{R}$ are defined by:

$$
\begin{aligned}
& \overline{D^{-} f}(x ; v)=\liminf _{h \rightarrow 0^{+}, v^{\prime} \rightarrow v} \frac{f\left(x+h v^{\prime}\right)-f(x)}{h} \\
& \overline{D^{+} f}(x ; v)=\limsup _{h \rightarrow 0^{+}, v^{\prime} \rightarrow v} \frac{f\left(x+h v^{\prime}\right)-f(x)}{h} .
\end{aligned}
$$


If $f$ is locally Lipschitz, one may notice that

$$
\overline{D^{+} f}(x ; v)=\limsup _{h \rightarrow 0^{+}} \frac{f(x+h v)-f(x)}{h}
$$

and if $f$ is continuously differentiable, then

$$
\overline{D^{+} f}(x ; v)=\langle\nabla f(x), v\rangle,
$$

(see (Smirnov 2002)).

\section{Asymptotic and finite time stability}

Let $\mathcal{X}$ be an open set of $\mathbb{R}^{n}$ containing the origin, $F$ a set valued function on $\mathcal{X} . F$ defined the differential inclusion

$$
\dot{x} \in F(x), \quad x \in \mathcal{X} .
$$

where $\dot{x}$ denotes the right derivatives of $x$. A solution $\phi(t)$ of the system (2) is an absolutely continuous function defined on an interval and satisfying the inclusion almost everywhere (that is $\dot{\phi}(t) \in F(\phi(t)$ ) holds almost everywhere in $t$ ). One define general conditions to have an existence theorem for solutions of differential inclusion:

Condition 1 (Filippov 1988) Let $F$ be a set valued function on $\mathcal{X}$. F satisfy the general conditions if:

(i) for all $x \in \mathcal{X}$, the set $F(x)$ is non empty, closed and convex,

(ii) the function $x \mapsto F(x)$ is upper semi-continuous.

By virtue of the general theorem of existence for solutions of differential inclusions in (Filippov 1988), (Aubin \& Cellina 1984), conditions 1 are sufficient for the existence for $x_{0} \in \mathcal{X}$ of a solution $\phi(t)$ of the system (2), defined on an interval $[0, d]$ with $d>0$, such that $\phi(0)=x_{0}$. Afterwards, $\phi^{x_{0}}(t)$ will represent a solution of the system (2) starting from $x_{0}$ and $\mathcal{S}\left(x_{0}\right)$ the set of all solutions $\phi^{x_{0}}$. We shall supply the space $\mathcal{S}=\bigcup_{x_{0} \in \mathcal{X}} \mathcal{S}\left(x_{0}\right)$ included in $C([0,+\infty[, \mathcal{X})$ with the topology of uniform convergence.

Now, one may introduce the notion of stability for a differential inclusion. Let us consider the system (2) with $F$ a set valued function such that $0 \in F(0)$. The origin is stable for the system (2) if for each $\epsilon>0$, there exists $\delta(\epsilon)>0$ such that if $x_{0} \in \delta(\epsilon) \mathcal{B}$ then each solution $\phi^{x_{0}}(t)$ exists for $t \geq 0$, and satisfies $\phi^{x_{0}}(t) \in \epsilon \mathcal{B}$ for all $t \geq 0$.

The origin is asymptotically stable for the system (2) if:

1. the origin is stable for the system (2), 
2. the origin is attractive, i.e. for each $\epsilon>0$, there exists $\delta(\epsilon)>0$ such that if $x_{0} \in \delta(\epsilon) \mathcal{B}$ then each solution $\phi^{x_{0}}(t)$ satisfies $\lim _{t \rightarrow \infty} \phi^{x_{0}}(t)=0$.

Now, one can give a non autonomous version of Lyapunov's theorem starting from a general non autonomous system:

Theorem 2 Let $F$ be a set valued function on $\mathcal{X}$ satisfying the general conditions 1 such that $0 \in F(0)$. If there exists a continuous positive definite function $V: \mathcal{V} \rightarrow \mathbb{R}_{\geq 0}$ and $W: \mathbb{R}^{n} \rightarrow \mathbb{R}$ a negative definite function such that:

$$
\overline{D^{+} V}(x ; v) \leq W(x), \quad \forall v \in F(x)
$$

for all $x \in \mathcal{V}$, then the origin of the system (2) is asymptotically stable. $V$ is called $a$ Lyapunov function for the system (2).

This theorem and its proof can be found in (Smirnov 2002, Theorem 8.1 and 8.2 ).

With the asymptotic stability, solutions of the system (2) tends to the origin. But, this concept lacks of information concerning the time convergence to the origin. To overcome this, the notion of finite time stability is introduced.

Definition 3 The origin is finite time stable for the system (2) if:

1. the origin is stable for the system (2): for all $\epsilon>0$, there is $\delta(\epsilon)>0$ such that if $x_{0} \in \delta(\epsilon) \mathcal{B}$, then $\phi^{x_{0}}$ is defined for all $t \geq 0$ and $\phi^{x_{0}}(t) \in \epsilon \mathcal{B}$ for all $t \geq 0$,

2. there exists $T_{0}: \mathcal{S} \rightarrow \mathbb{R}_{\geq 0}$, such that for all solutions $\phi^{x_{0}} \in \mathcal{S}\left(x_{0}\right)$, $\phi^{x_{0}}(t)=$ 0 for all $t \geq T_{0}\left(\phi^{x_{0}}\right)$. $T_{0}$ is the settling time of the system (2).

The notion of finite time stability is illustrated by figure 1 .

One may even give the following result.

Proposition 4 Consider the system (2) where $F$ satisfies the condition 1 and is Lipschitzean with compact values outside the origin, if the system (2) is finite time stable on $\delta(\epsilon) \mathcal{B}$ with a continuous settling time $T_{0}$, then for all $x \in \delta(\epsilon) \mathcal{B}$, $T(x)=\sup _{\phi^{x} \in \mathcal{S}(x)} T_{0}\left(\phi^{x}\right)<+\infty . T(x)$ is called the settling time with respect to initial conditions of the system (2).

Proof. Suppose that the system (2) is finite time stable with a continuous settling time $T_{0}$. As the system is Lipschitzean with compact values, the following result (Aubin \& Frankowska 1990, Corollary 10.4.5) ensures that $\mathcal{S}(x)$ is dense in $\tilde{\mathcal{S}}(x)$ for the uniform convergence where $\tilde{\mathcal{S}}(x)$ is the set of solutions of the system

$$
\dot{x} \in \overline{c o} F(x), \quad x \in \mathcal{X} \backslash\{0\} .
$$




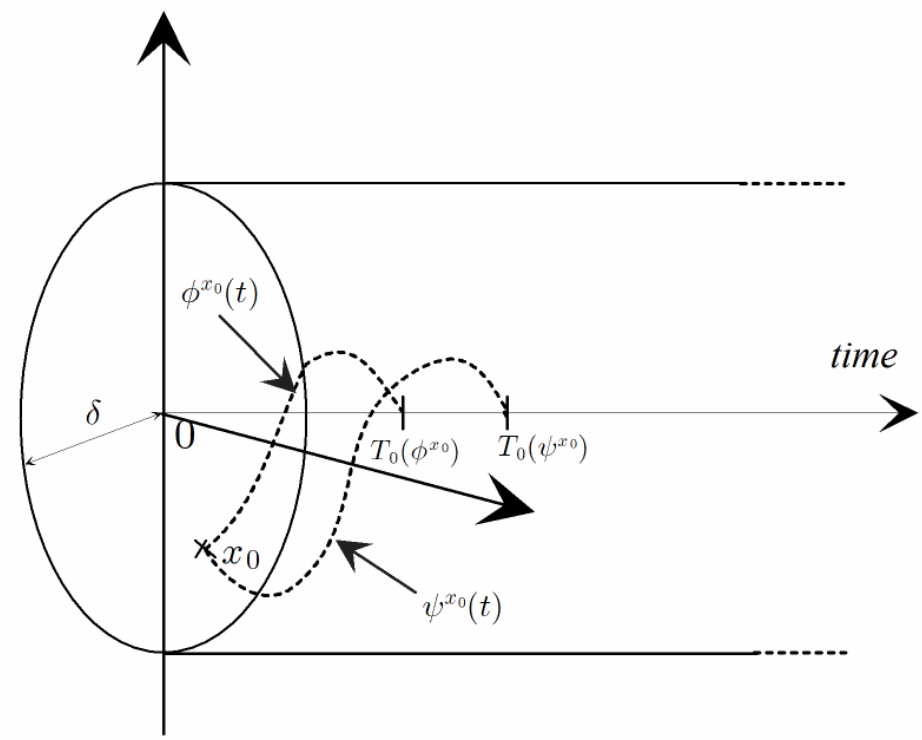

Figure 1: Finite time stability

As $F$ satisfies the condition 1, $F(x)=\overline{c o} F(x)$ and then $\mathcal{S}(x)$ is closed for $x \in$ $\mathcal{X} \backslash\{0\}$. Suppose that there exists $x_{0} \in \delta(\epsilon) \mathcal{B} \backslash\{0\}$ such that $\sup _{\phi^{x_{0}} \in \mathcal{S}\left(x_{0}\right)} T_{0}\left(\phi^{x_{0}}\right)=$ $+\infty$. Then there exists a sequence $\phi_{n}^{x_{0}}$ of solutions in $\mathcal{S}\left(x_{0}\right)$ such that

$$
\lim _{n \rightarrow+\infty} T_{0}\left(\phi_{n}^{x_{0}}\right)=+\infty
$$

But $\lim _{n \rightarrow+\infty} \phi_{n}^{x_{0}}$ belongs to $\mathcal{S}\left(x_{0}\right)$. By assumption $T_{0}\left(\lim _{n \rightarrow+\infty} \phi_{n}^{x_{0}}\right)<+\infty$. As $T_{0}$ is continuous, $\lim _{n \rightarrow+\infty} T_{0}\left(\phi_{n}^{x_{0}}\right)=T_{0}\left(\lim _{n \rightarrow+\infty} \phi_{n}^{x_{0}}\right)$, which is a contradiction. Moreover, $T_{0}(0)=0$ because of the stability of the system (2).

\section{Sufficient conditions for finite time stability}

In this section, we extend a result coming from (Haimo 1986) to differential equations. For a Lyapunov function $V$ one needs the following inequality

$$
\overline{D^{+} V}(x ; v) \leq-r(V(x))
$$

for all $x \in \mathcal{V}$ and all $v \in F(x)$ where $r: \mathbb{R}_{\geq 0} \rightarrow \mathbb{R}_{\geq 0}$ is continuous and satisfies $r(0)=0$. 
Since the use of a Lyapunov function will lead to some scalar differential inequalities, the following proposition will give a sufficient condition for finite time stability: the existence of a Lyapunov function satisfying the condition (3).

\subsection{Use of smooth Lyapunov functions}

Proposition 5 Let the system (2) with $0 \in F(0)$. If there exists a continuously differentiable Lyapunov function satisfying condition (3) with a positive definite continuous function $r: \mathbb{R}_{\geq 0} \rightarrow \mathbb{R}_{\geq 0}$ such that for all $\epsilon>0$

$$
\int_{0}^{\epsilon} \frac{d z}{r(z)}<+\infty
$$

then the origin of the system (2) is finite time stable.

Proof. Since $V: \mathcal{V} \rightarrow \mathbb{R}_{\geq 0}$ is a Lyapunov function, then the origin is asymptotically stable (see Theorem 2). Let $\phi^{x_{0}}(t)$ be a solution of (2) which tends to the origin with the settling time $T_{0}\left(\phi^{x_{0}}\right)\left(0 \leq T_{0}\left(\phi^{x_{0}}\right) \leq+\infty\right.$ : from the attractivity of the origin). It remains to be proven that $T_{0}\left(\phi^{x_{0}}\right)<+\infty$. Using the asymptotic stability definition, $x_{0}$ can be chosen small enough to ensure that $\phi^{x_{0}}(t) \in \mathcal{V}$ for $t \geq 0$ and $t \mapsto V\left(\phi^{x_{0}}(t)\right)$ strictly decreases for $\tau \geq t$. Using the change of variables: $\left[0, T_{0}\left(\phi^{x_{0}}\right)\right] \rightarrow\left[0, V\left(x_{0}\right)\right]$ given by $z=V\left(\phi^{x_{0}}(t)\right)$, one obtains

$$
\int_{V\left(x_{0}\right)}^{0} \frac{d z}{-r(z)}=\int_{0}^{T_{0}\left(\phi^{x_{0}}\right)} \frac{\left\langle\nabla V\left(\phi^{x_{0}}(t)\right), \dot{\phi}^{x_{0}}(t)\right\rangle}{-r\left(V\left(\phi^{x_{0}}(t)\right)\right)} d t .
$$

Since $V$ is continuously differentiable then, by (1), condition (3) reads as

$$
\langle\nabla V(x), v\rangle \leq-r(V(x))
$$

for all $x \in \mathcal{V}$ and all $v \in F(x)$. This shows that

$$
T_{0}\left(\phi^{x_{0}}\right)=\int_{0}^{T_{0}\left(\phi^{x_{0}}\right)} d t \leq \int_{0}^{V\left(x_{0}\right)} \frac{d z}{r(z)} .
$$

This implies that $T_{0}\left(\phi^{x_{0}}\right)<+\infty$. Furthermore, $\int_{0}^{V\left(x_{0}\right)} \frac{d z}{r(z)}$ is independent of $\phi^{x_{0}}$, this shows that for any solutions $\phi^{x_{0}}(t)$ initiated at $x_{0}$ we have $T\left(x_{0}\right) \leq$ $\int_{0}^{V\left(x_{0}\right)} \frac{d z}{r(z)}<+\infty$. Thus, the origin of the system (2) is finite time stable.

Remark 6 The settling time with respect to initial conditions of the system (2) satisfies the following inequality

$$
T(x) \leq \int_{0}^{V(x)} \frac{d z}{r(z)}
$$

so it is continuous at the origin. 
Example 7 Let us consider the set valued function

$$
F(x)=\left\{\begin{array}{lll}
1+|x|^{\frac{1}{2}} & \text { if } x<0 \\
{[-1,1]} & \text { if } x=0 \\
-1-|x|^{\frac{1}{2}} & \text { if } x>0
\end{array}\right.
$$

and the system $\dot{x} \in F(x)$. Let $V(x)=x^{2}$, then for all $v \in F(x)$

$$
\langle\nabla V(x), v\rangle \leq-|x|^{\frac{3}{2}}=-V(x)^{\frac{3}{4}} .
$$

So the origin of the system is finite time stable.

Differential inclusion is one of the most interesting tools for studying differential equations with discontinuous righthand sides ( $\operatorname{such}$ as $\dot{x} \underset{a . e}{=} f(x)$ ). So, one considers a function $f: \mathcal{X} \rightarrow \mathbb{R}^{n}$ satisfying the conditions:

- $f$ is measurable on $\mathcal{X}$,

- $f$ is locally with relative compact value ${ }^{1}$,

- $f(0)=0$.

One defines for all $x \in \mathcal{X}$ the set valued function $\mathcal{F}$ by:

$$
\mathcal{F}(x)=\bigcap_{\epsilon>0} \overline{c o}(f(x+\epsilon \overline{\mathcal{B}})) .
$$

For all $x \in \mathcal{X}$, the set $\mathcal{F}(x)$ is non empty, bounded, closed and convex. Moreover, the set valued function $\mathcal{F}$ can be easily shown to be upper semi-continuous in $x$, so $\mathcal{F}$ satisfy the general conditions 11. Thus the previous theory may be applied to the following differential inclusion:

$$
\dot{x} \in \mathcal{F}(x), \quad x \in \mathcal{X} .
$$

A solution of the system (6) is called a Krasovskii solution. Let us give an example of finite time stable discontinuous system.

Example 8 Let the system

$$
\left\{\begin{array}{l}
\dot{x}_{1}=-\operatorname{sgn}\left(x_{1}\right)-x_{1}^{3}+x_{2} \\
\dot{x}_{2}=-\operatorname{sgn}\left(x_{2}\right)-x_{2}^{3}-x_{1}
\end{array}\right.
$$

where sgn is the function defined on $\mathbb{R}$ by

$$
\operatorname{sgn}(x)=\left\{\begin{array}{cll}
-1 & \text { if } & x<0 \\
1 & \text { if } & x \geq 0
\end{array} .\right.
$$

\footnotetext{
${ }^{1} f: \mathcal{X} \rightarrow \mathbb{R}^{n}$ is locally with relative compact value if for all compact $\mathcal{K}$ of $\mathcal{X}$, there exists $\delta(\mathcal{K})>0$ such that for all $\delta<\delta(\mathcal{K}), \overline{f(x+\delta \mathcal{B})}$ is compact in $\mathbb{R}^{n}$.
} 
Then the associated differential inclusion to system (7) is

$$
\left\{\begin{array}{c}
\dot{x}_{1} \in-\mathcal{S G \mathcal { N }}\left(x_{1}\right)-x_{1}^{3}+x_{2} \\
\dot{x}_{2} \in-\mathcal{S G N}\left(x_{2}\right)-x_{2}^{3}-x_{1}
\end{array}\right.
$$

where

$$
\mathcal{S G N}(x)=\left\{\begin{array}{cll}
-1 & \text { if } & x<0 \\
{[-1,1]} & \text { if } & x=0 \\
1 & \text { if } & x>0
\end{array} .\right.
$$

is a set valued function. Taking $V(x)=\frac{\|x\|^{2}}{2}$, one obtains for all $v \in\left(-\mathcal{S G N}\left(x_{1}\right)-x_{1}^{3}+x_{2},-\mathcal{S} \mathcal{G N}\left(x_{2}\right)-x_{2}^{3}-x_{1}\right)$

$$
\langle\nabla V(x), v\rangle=-\sum_{i=1}^{2}\left(x_{i}^{4}+\left|x_{i}\right|\right) \leq 0 .
$$

Then, $V$ is a Lyapunov function for the system (7). As $\sum_{i=1}^{2}\left(x_{i}^{4}+\left|x_{i}\right|\right) \geq$ $\left(x_{1}^{2}+x_{2}^{2}\right)^{\frac{1}{2}}=\|x\|$, one deduces that $\langle\nabla V(x), v\rangle \leq-\sqrt{2} V(x)^{\frac{1}{2}}$. Using the proposition 5, one deduces that the origin of system (7) is finite time stable with the settling time $T(x) \leq\|x\|$.

Finally, one may notice that the system of the example (7) may be defined using differential equation with discontinuous righthand sides as $\dot{x}=-\operatorname{sgn}(x)-$ $|x|^{\frac{1}{2}} \operatorname{sgn}(x)$.

\subsection{Use of nonsmooth Lyapunov functions}

When dealing with nonsmooth Lyapunov functions, we shall use the following comparison lemma:

Lemma 9 (Comparison lemma) If the scalar differential equation

$$
\dot{x}=f(x), x \in J \subset \mathbb{R},
$$

has the global semi-flow $\Phi: \mathbb{R}_{\geq 0} \times J \rightarrow \mathbb{R}$, where $f$ is locally Lipschitz, and if $g:[a, b) \rightarrow \mathbb{R}$ (b could be infinity) is a continuous function such that

$$
\overline{D^{+} g}(t ; 1) \leq f(g(t)), \quad t \in[a, b),
$$

then $g(t) \leq \Phi(t, g(a))$ for all $t \in[a, b)$.

This result comes from a slight modification of (Smirnov 2002, lemma 8.1) and (Hassan 1996, lemma 3.4). Now, one can give a sufficient condition for finite time stability. 
Proposition 10 Let the system (2) with $0 \in F(0)$. If there exists a Lyapunov function for the system (2) satisfying condition (3) with a positive definite continuous function $r: \mathbb{R}_{\geq 0} \rightarrow \mathbb{R}_{\geq 0}$ locally Lipschitz outside the origin such that

$$
\int_{0}^{\epsilon} \frac{d z}{r(z)}<+\infty
$$

for all $\epsilon>0$, then the origin is finite time stable. Moreover, the settling time with respect to initial conditions of the system (2) satisfies:

$$
T(x) \leq \int_{0}^{V(x)} \frac{d z}{r(z)} .
$$

If in addition, $V$ is decrescent then the origin is uniformly finite time stable.

Proof. Since $V: \mathcal{V} \rightarrow \mathbb{R}_{\geq 0}$ is Lyapunov function of the system (2) satisfying condition (10), then from theorem 2, the origin is asymptotically stable. Let $x_{0} \in \mathcal{V}$ and $\phi^{x_{0}}(t)$ be a solution of (2) which tends to the origin with the settling time $T_{0}\left(\phi^{x_{0}}\right)$. It remains to prove that $T_{0}\left(\phi^{x_{0}}\right)<+\infty$. Because of asymptotic stability, one may suppose with no loss of generality that $\phi^{x_{0}}(t) \in \mathcal{V}$ for $t \geq 0$. Let us consider the system

$$
\dot{z}=-r(z), \quad z \geq 0,
$$

with the global semi flow $\Phi(t, z)$ for $z \geq 0$. Using the proof of (Smirnov 2002, Theorem 8.1), one deduces that there exists $v \in F\left(\phi^{x_{0}}(t)\right)$ such that

$$
\overline{D^{+}\left(V \circ \phi^{x_{0}}\right)}(t ; 1)=\overline{D^{+} V}\left(\phi^{x_{0}}(t) ; v\right) \leq-r\left(V\left(\phi^{x_{0}}(t)\right)\right)
$$

for all $t \geq 0$ and all $x_{0} \in \mathcal{V} \backslash\{0\}$. Now, applying the comparison lemma 9, one deduces that

$$
V\left(\phi^{x_{0}}(t)\right) \leq \Phi\left(t, V\left(x_{0}\right)\right), \quad t \geq 0, x_{0} \in \mathcal{V} \backslash\{0\} .
$$

$>$ From (Haimo 1986) and (Moulay \& Perruquetti 2003), one knows that

$$
\Phi(t, z)=0 \text { for } t \geq \int_{0}^{V(x)} \frac{d z}{r(z)} .
$$

With the positive definiteness of $V$, one concludes that

$$
\phi^{x_{0}}(t)=0 \text { for } t \geq \int_{0}^{V(x)} \frac{d z}{r(z)} .
$$

Thus, the origin of the system (2) is finite time stable. Moreover, $\int_{0}^{V(x)} \frac{d z}{r(z)}$ is independent of $\phi^{x_{0}}$, this shows that $T\left(x_{0}\right)<+\infty$.

Remark 11 One may notice that if condition (3) is an equality for all $x \in$ $\mathcal{V}$ and all $v \in F(x)$, then the conclusion of the proposition (10) is that all solutions in $\mathcal{S}(x)$ reach the origin with the same settling time with respect to initial conditions of the system (2) $T(x)=\int_{0}^{V(x)} \frac{d z}{r(z)}$. One remarks that this problem does not set for systems with uniqueness of solutions outside the origin. 
Now, we want to give an example using a only continuous Lyapunov function.

Example 12 Let $0<a<1$, and consider the system

$$
\left\{\begin{array}{l}
\dot{x}_{1}=-\left|x_{1}\right|^{\frac{1}{2}} \operatorname{sgn}\left(x_{1}\right)+\left|x_{2}\right| \\
\dot{x}_{2}=-\operatorname{sgn}\left(x_{2}\right)
\end{array}\right.
$$

The differential inclusion associated to (9) is

$$
\left\{\begin{array}{l}
\dot{x}_{1} \in-\left|x_{1}\right|^{\frac{1}{2}} \operatorname{sgn}\left(x_{1}\right)+\left|x_{2}\right| \\
\dot{x}_{2} \in-\mathcal{S G N}\left(x_{2}\right)
\end{array} .\right.
$$

Where $\mathcal{S G N}$ is defined by (8). Let us consider the following continuous function $V(x)=\left|x_{1}\right|+x_{2}^{2}$. For all $v \in\left(-\left|x_{1}\right|^{\frac{1}{2}} \operatorname{sgn}\left(x_{1}\right)+\left|x_{2}\right|,-\mathcal{S G N}\left(x_{2}\right)\right)$, the following holds

$$
\begin{aligned}
\overline{D^{+} V}(x ; v) & =-\left|x_{1}\right|^{\frac{1}{2}}+\left|x_{2}\right| \operatorname{sgn}\left(x_{1}\right)-2\left|x_{2}\right| \\
& \leq-\left(\left|x_{1}\right|^{\frac{1}{2}}+\left|x_{2}\right|\right) \leq-V(x)^{\frac{1}{2}} .
\end{aligned}
$$

So the system is finite time stable.

Let us sum up the two previous results: in order to show finite time stability, we have the choice between a pair $\left(V_{1}, r_{1}\right)$ with $V_{1}$ which is continuously differentiable and $r_{1}$ only continuous, or a pair $\left(V_{2}, r_{2}\right)$ with $V_{2}$ which is continuous and $r_{2}$ locally Lipschitz. Moreover, the two conditions are not equivalent because there is no converse theorem for the finite time stability of differential inclusions. Moreover, because of the regularity of $r$, the proposition 10 does not imply that there exists a smooth Lyapunov function satisfying the assumptions of the proposition 5. So the proposition 5 is not weaker than the proposition 10. These two results are complementary.

\section{Necessary conditions}

Now, for a smooth Lyapunov function $V$ one needs the following inequality

$$
\overline{D^{-} V}(x ; v) \geq-s(V(x))
$$

for all $x \in \mathcal{V}$ and all $v \in F(x)$ where $s: \mathbb{R}_{\geq 0} \rightarrow \mathbb{R}_{\geq 0}$ is continuous and satisfies $s(0)=0$.

\subsection{Use of smooth Lyapunov functions}

Proposition 13 Let the system (2) with $0 \in F(0)$, if the origin is finite time stable for the system (2) then for all Lyapunov functions satisfying condition (10) with a positive definite continuous function $s: \mathbb{R}_{\geq 0} \rightarrow \mathbb{R}_{\geq 0}$,

$$
\int_{0}^{\epsilon} \frac{d z}{s(z)}<+\infty
$$


for all $\epsilon>0$.

Proof. Suppose that $V: \mathcal{V} \rightarrow \mathbb{R}_{>0}$ is a smooth Lyapunov function satisfying condition (10). Let $\phi^{x_{0}}(t)$ be a solution of (2) with the settling time $0 \leq$ $T_{0}\left(\phi^{x_{0}}\right)<+\infty$. Because of the asymptotic stability, one may choose $x_{0}$ small enough to ensure that $\phi^{x_{0}}(t) \in \mathcal{V}$ for all $t \geq 0$ and $t \mapsto V\left(\phi^{x_{0}}(t)\right)$ strictly decreases for $t \geq 0$. Using the change of variables, $\left[0, T_{0}\left(\phi^{x_{0}}\right)\right] \rightarrow\left[0, V\left(x_{0}\right)\right]$ given by $z=V\left(\phi^{x_{0}}(t)\right)$, one obtains

$$
\int_{V\left(x_{0}\right)}^{0} \frac{d z}{-s(z)}=\int_{0}^{T_{0}\left(\phi^{x_{0}}\right)} \frac{\left\langle\nabla V\left(\phi^{x_{0}}(t)\right), \dot{\phi}^{x_{0}}(t)\right\rangle}{-s\left(V\left(\phi^{x_{0}}(t)\right)\right)} d t .
$$

Since $\langle\nabla V(x), v\rangle \geq-s(V(x))$ for all $x \in \mathcal{V}$ and all $v \in F(x)$ and $-s\left(V\left(\phi^{x_{0}}(t)\right)\right)<$ 0 for all $t \geq 0$ one obtains

$$
\int_{0}^{V\left(x_{0}\right)} \frac{d z}{s(z)} \leq \int_{0}^{T_{0}\left(\phi^{x_{0}}\right)} d t=T_{0}\left(\phi^{x_{0}}\right)<+\infty .
$$

This proposition may be used to show the non finite time stability as follow.

Example 14 Let us consider the system

$$
\left\{\begin{array}{l}
\dot{x}_{1}=\left|x_{2}\right|-x_{1}^{3} \\
\dot{x}_{2}=-x_{1} \operatorname{sgn}\left(x_{2}\right)-x_{2}^{3}
\end{array}\right.
$$

Let $V(x)=\frac{x_{1}^{2}+x_{2}^{2}}{2}$, for all $v \in\left(\left|x_{2}\right|-x_{1}^{3},-x_{1} \mathcal{S G N}\left(x_{2}\right)-x_{2}^{3}\right)$ one obtains

$$
\langle\nabla V(x), v\rangle=-\left(x_{1}^{4}+x_{2}^{4}\right) .
$$

Then, $V$ is a Lyapunov function for the system such that

$$
\langle\nabla V(x), v\rangle \geq-\left(x_{1}^{4}+x_{2}^{4}\right) \geq-4 V(x)^{2} .
$$

Since

$$
\int_{0}^{\epsilon} \frac{d z}{z^{2}}=+\infty
$$

for all $\epsilon>0$, the proposition 13 ensures that the origin is not finite time stable.

\subsection{Use of nonsmooth Lyapunov functions}

Proposition 15 Let the system (2) with $0 \in F(0)$. If the origin is finite time stable for the system (2) then for all Lyapunov function satisfying condition (10) with with a positive definite continuous function $s: \mathbb{R}_{\geq 0} \rightarrow \mathbb{R}_{\geq 0}$ locally Lipschitz outside the origin,

$$
\int_{0}^{\epsilon} \frac{d z}{s(z)}<+\infty
$$

for all $\epsilon>0$. 
Proof. Suppose that $V$ is a Lyapunov function satisfying condition (10) with a positive definite continuous function $s: \mathbb{R}_{>0} \rightarrow \mathbb{R}_{>0}$ locally Lipschitz outside the origin $s$. Let $x_{0} \in \mathcal{V}$ and $\phi^{x_{0}}(t)$ be a solution of (2) with the settling time $0 \leq T_{0}\left(\phi^{x_{0}}\right)<+\infty$. Because of the asymptotic stability, one may suppose with no loss of generality that $\phi^{x_{0}}(t) \in \mathcal{V}$ for all $t \geq 0$. Let us consider the system

$$
\dot{z}=-s(z), \quad z \geq 0,
$$

with the global semi flow $\Phi(t, z)$ for $z \geq 0$. Using the proof in (Smirnov 2002, Theorem 8.1), one deduces that there exists $v \in F\left(\phi^{x_{0}}(t)\right)$ such that

$$
\overline{D^{+}\left(V \circ \phi^{x_{0}}\right)}(t ; 1) \geq \overline{D^{-}\left(V \circ \phi^{x_{0}}\right)}(t ; 1)=\overline{D^{-} V}\left(\phi^{x_{0}}(t) ; v\right) \geq-s\left(V\left(\phi^{x_{0}}(t)\right)\right)
$$

for all $t \geq 0$ and all $x_{0} \in \mathcal{V} \backslash\{0\}$. Now, applying the comparison lemma (9), one deduces that

$$
V\left(\phi^{x_{0}}(t)\right) \geq \Phi\left(t, V\left(x_{0}\right)\right) \geq 0, \quad t \geq 0, x_{0} \in \mathcal{V} \backslash\{0\}
$$

As the origin of the system (2) is finite time stable, one deduces that

$$
\Phi(t, z)=0 \text { for } z \geq 0 \text { and } t \geq T_{0}\left(\phi^{z}\right) .
$$

So, the system (11) is finite time stable. From (Haimo 1986) and (Moulay \& Perruquetti 2003), one deduces that for all $\epsilon>0$,

$$
\int_{0}^{\epsilon} \frac{d z}{s(z)}<+\infty
$$

One more time, we have the choice between a pair $\left(V_{1}, s_{1}\right)$ with $V_{1}$ which is continuously differentiable and $s_{1}$ only continuous, or a pair $\left(V_{2}, s_{2}\right)$ with $V_{2}$ which is continuous and $s_{2}$ locally Lipschitz to show the non finite time stability. The two conditions are not equivalent because of the regularity of $s$.

\section{Conclusion}

Finite time stability for differential inclusions is investigated using Lyapunov functions. Indeed, these functions are at the center of stability problems. It is a powerful tool which allows to solve a part of the finite time stability problem. For the moment, the Lyapunov functions does not give a necessary and sufficient condition (only two necessary conditions and two sufficient ones). This has been done using two ways: the first one with integration properties and smooth Lyapunov functions and the second one with a comparison lemma and nonsmooth Lyapunov functions.

Nevertheless, it is enough to investigate finite time stabilization using discontinuous controller which is at the heart of many researches on control theory. Thus, the following step will be to develop the finite time stabilization using discontinuous feedback. 


\section{References}

Aubin, J. P. \& Cellina, A. (1984), Differential Inclusion, Vol. 264, Grundlehren der mathematischen Wiissenschaften.

Aubin, J. P. \& Frankowska, H. (1990), Set-Valued Analysis, Springer-Verlag, New-York.

Bhat, S. P. \& Bernstein, D. S. (2000), 'Finite time stability of continuous autonomous systems', SIAM J. Control Optim. 38(3), 751-766.

Filippov, A. F. (1988), Differential Equations with Discontinuous Righthand Sides, Kluwer Academic Publishers, Dordrecht/Boston/London.

Haimo, V. T. (1986), 'Finite time controllers', SIAM J. Control and Optimisation 24(4), 804-833.

Hassan, K. K. (1996), Nonlinear Systems, NJ 07458, Prentice-Hall, Upper Saddle River.

Hong, Y. (2002), 'Finite-time stabilization and stabilizability of a class of controllable systems', Systems and Control Letters 46, 231-236.

Moulay, E. \& Perruquetti, W. (2003), Finite time stability of non linear systems, in 'IEEE Conference on Decision and Control', Maui, Hawaii, pp. 36413646 .

Orlov, Y. (2003), Finite time stability of homogeneous switched systems, in 'IEEE Conference on Decision and Control', pp. 4271-4276.

Perruquetti, W. \& Drakunov, S. (2000), Finite time stability and stabilisation, in 'IEEE Conference on Decision and Control', Sydney (Australia).

Smirnov, G. V. (2002), Introduction to the Theory of Differential Inclusions, Vol. 41, Graduate Studies in Mathematics. 